\title{
ÍNDICE DE VULNERABILIDADE SOCIAL: UMA APLICAÇÃO DE ANÁLISE FATORIAL PARA CLASSIFICAR OS MUNICÍPIOS PERNAMBUCANOS ${ }^{1}$
}

Cláudia César Batista Julião²

João Eustáquio de Lima

0 entendimento de situações tradicionalmente definidas como de pobreza tem sido progressivamente substituído por discussões para além de sua dimensão estritamente monetária, passando a considerar noções de exclusão e vulnerabilidade social. Nesse sentido, este trabalho tem como objetivo classificar os municípios pernambucanos pelo índice de vulnerabilidade social (IVS), que será construído a partir da técnica multivariada de análise fatorial (AF) para o ano de 2010. Além disso, o estudo visa comparar os resultados do IVS calculado pela análise fatorial com os do IVS já desenvolvido pelo Ipea e baseado em outra metodologia. Em termos gerais, a partir da análise, observou-se que o valor médio do IVS AF para os municípios pernambucanos, em 2010, foi de 0,520, e teve um valor mais alto do que o IVS Ipea, que foi de 0,470. Assim, este resultado sugere que a técnica multivariada de análise fatorial pode ter contribuído para refletir melhor as situações de vulnerabilidade dos municípios pernambucanos e não subestimar a vulnerabilidade social nestes.

Palavras-chave: vulnerabilidade social; análise fatorial; Pernambuco.

\section{SOCIAL VULNERABILITY INDEX: A FACTOR ANALYSIS APPLICATION TO CLASSIFY CITIES OF PERNAMBUCO}

The understanding of situations traditionally defined as poverty has been progressively replaced by discussions beyond its strictly monetary dimension, starting to consider notions of exclusion and social vulnerability. In this sense, this study aims to classify the municipalities of Pernambuco by the social vulnerability index (IVS), which will be constructed using the multivariate technique of factorial analysis for the year of 2010. In addition, the present study aims to compare the results of the calculated IVS by the factorial analysis with those of IVS already developed by Ipea and based on another methodology. In general terms, from the analysis, it was observed that the mean value of IVS AF for the municipalities of Pernambuco in 2010 was 0.520 and had a higher value than IVS Ipea that was 0.470 . Thus, this result suggests that the multivariate technique of factor analysis may have contributed to better reflect the vulnerability situations of the municipalities of Pernambuco and not to underestimate their social vulnerability.

Keywords: social vulnerability; factor analysis; Pernambuco. 


\section{ÍNDICE DE VULNERABILIDAD SOCIAL: UNA APLICACIÓN DE ANÁLISIS DE FACTORES PARA CLASIFICAR MUNICIPIOS DE PERNAMBUCO}

La comprensión de situaciones tradicionalmente definidas como pobreza ha sido reemplazada progresivamente por discusiones más allá de su dimensión estrictamente monetaria, comenzando a considerar las nociones de exclusión y vulnerabilidad social. En este sentido, este trabajo tiene como objetivo clasificar los municipios de Pernambuco por el índice de vulnerabilidad social (SIV) que se construirá utilizando la técnica multivariada de análisis factorial para el año 2010. Además, el presente estudio tiene como objetivo comparar los resultados del SVI calculado por análisis factorial con los del IVS ya desarrollado por Ipea y basado en otra metodología. En términos generales, a partir del análisis, se observó que el valor promedio del IVS AF para los municipios de Pernambuco, en 2010, era 0.520 y tenía un valor más alto que el IVS Ipea, que era 0.470 . Por lo tanto, este resultado sugiere que la técnica de análisis factorial multivariante puede haber contribuido a reflejar mejor las situaciones de vulnerabilidad de los municipios de Pernambuco y no a subestimar su vulnerabilidad social.

Palabras clave: vulnerabilidad social; análisis factorial; Pernambuco.

\section{INDICE DE VULNÉRABILITÉ SOCIALE: UNE APPLICATION D'ANALYSE DE FACTEUR POUR CLASSIFIER LES MUNICIPALITÉS DE PERNAMBUCO}

La compréhension des situations traditionnellement définies comme la pauvreté a été progressivement remplacée par des discussions au-delà de sa dimension strictement monétaire, en commençant à considérer les notions d'exclusion et de vulnérabilité sociale. En ce sens, ce travail vise à classer les communes de Pernambuco par l'indice de vulnérabilité sociale (IVS) qui sera construit en utilisant la technique multivariée de l'analyse factorielle pour l'année 2010. De plus, la présente étude vise à comparer les résultats de l'IVS calculé par analyse factorielle avec celles de I'IVS déjà développées par Ipea et basées sur une autre méthodologie. De manière générale, à partir de l'analyse, il a été observé que la valeur moyenne de l'IVS AF pour les municipalités de Pernambuco, en 2010, était de 0,520 et avait une valeur plus élevée que I'IVS Ipea, qui était de 0,470 . Ainsi, ce résultat suggère que la technique d'analyse factorielle multivariée peut avoir contribué à mieux refléter les situations de vulnérabilité des municipalités de Pernambuco et à ne pas sous-estimer leur vulnérabilité sociale.

Mots-clés: vulnérabilité sociale; analyse factorielle; Pernambuco.

JEL: 132.

\section{INTRODUÇÃO}

O entendimento de situaçóes tradicionalmente definidas como de pobreza tem sido progressivamente substituído por discussóes para além de sua dimensão estritamente monetária, passando a considerar noçôes de exclusão e vulnerabilidade social. Pesquisadores e operadores de políticas sociais, cada vez mais, buscam ampliar a perspectiva de pobreza atrelada apenas à questão da insuficiência de renda (Costa e Marguti, 2015). 
Segundo Plesa (2013), o conceito de exclusão social é mais recente e mais abrangente do que o conceito de pobreza, visto que considera não apenas a falta de bens materiais, mas também a impossibilidade de inclusão em redes sociais, econômicas, políticas e culturais. Assim, o conceito de exclusão social envolve a escassez da participação em atividades sociais no dia a dia, além do deficit de renda considerado no conceito de pobreza.

Para Balan (2013), a vulnerabilidade social pode ser definida como uma situação de vida caracterizada por uma combinação multidimensional de vantagens e desvantagens, de fatores de inclusão e exclusão. Em oposição à noção de pobreza, vulnerabilidade social inclui aspectos que estâo associados não apenas com o nível de renda, mas também com as condiçôes de habitação, de mercado de trabalho e de cuidados destinados a crianças e pessoas dependentes.

Assim, dada a natureza multidimensional da vulnerabilidade social e a abrangência do seu escopo, evidencia-se ainda mais o papel do Estado na promoção do bem-estar da população. Nesse sentido, com a finalidade de auxiliar os gestores de políticas públicas, o Ipea desenvolveu um IVS para os municípios brasileiros como instrumento de identificação das falhas de oferta de bens e serviços públicos.

O referido IVS sinaliza o acesso, a ausência ou a insuficiência de ativos cuja posse ou privação determina as condições de bem-estar da população. Além disso, o índice é composto por dezesseis variáveis - dos Censos Demográficos do Instituto Brasileiro de Geografia e Estatística (IBGE) -, relacionadas a três dimensôes (Costa e Marguti, 2015), conforme a seguir descrito.

1) Infraestrutura urbana: dimensão que contempla variáveis de vulnerabilidade de infraestrutura urbana e procura refletir as condiçóes de acesso aos serviços de saneamento básico e de mobilidade urbana, dois aspectos relacionados ao lugar de domicílio das pessoas e que impactam significativamente no bem-estar.

2) Capital humano: dimensão que abrange variáveis referentes a capital humano e envolve dois aspectos que determinam as perspectivas (atuais e futuras) de inclusão social dos indivíduos: saúde e educação. Nesse sentido, as variáveis retratam não só a presença atual destes ativos e recursos nas populaçóes, mas também o potencial que suas geraçóes mais novas apresentam de ampliá-lo.

3) Renda e trabalho: dimensão que agrupa não só variáveis relativas à insuficiência de renda presente, mas incorpora outros fatores que, associados ao fluxo de renda, configuram um estado de insegurança de renda: a desocupação de adultos; a ocupação informal de adultos pouco escolarizados; a dependência com relação à renda de pessoas idosas; assim como a presença de trabalho infantil. 
Dessa forma, o IVS proposto pelo Ipea é calculado a partir da média aritmética de três subíndices que refletem as dimensóes citadas anteriormente. Metodologicamente, cada subíndice entra no cálculo do IVS com o mesmo peso, e, para as variáveis que os compóem, são deliberados pesos.

Não obstante, a despeito da elegância metodológica utilizada, há limitação desse índice no que se refere à arbitrariedade da seleçáo dos pesos utilizados. Assim, na tentativa de preencher essa lacuna, este trabalho tenta avançar com relação à metodologia, ao propor o uso da técnica multivariada de análise fatorial para a construção do índice de vulnerabilidade social.

Sendo assim, o objetivo deste trabalho é classificar os municípios pernambucanos pelo índice de vulnerabilidade social construído, para o ano de 2010, a partir da técnica multivariada de análise fatorial. Além disso, o estudo visa comparar os resultados do IVS calculado pela análise fatorial com os do IVS já estabelecido pelo Ipea.

A escolha de Pernambuco justifica-se por ser esta Unidade da Federação (UF) localizada no Nordeste, região brasileira que apresentou o segundo mais elevado índice de vulnerabilidade em 2010, segundo o Ipea. Além disso, nessa mesma classificação, Pernambuco revelou-se como o terceiro estado nordestino com maior concentração de municípios na faixa de muito alta vulnerabilidade social. Desse modo, é pertinente analisar mais categoricamente os municípios pernambucanos. O período de análise, por sua vez, justifica-se pela disponibilidade dos dados, que, apesar de serem extraídos da plataforma Atlas Brasil, são derivados do Censo Demográfico, sendo 2010 o último ano de recenseamento.

Além desta introdução, o trabalho está divido em mais quatro seções. Nas seçôes 2 e 3, são apresentadas, respectivamente, a estratégia empírica e a base de dados utilizadas a fim de se alcançar o objetivo proposto. A quarta seção dedica-se a expor os resultados obtidos na pesquisa. Por fim, na seção 5, são apresentadas as consideraçóes finais.

\section{ESTRATÉGIA EMPÍRICA}

A estratégia empírica adotada para atingir o objetivo deste trabalho foi o método de análise fatorial. Segundo Mingoti (2007), a análise fatorial é uma técnica estatística que tem como objetivo principal descrever a variabilidade original do vetor de variáveis em termos de um número menor de variáveis aleatórias chamadas de fatores comuns, os quais são variáveis latentes (não observadas) que representam uma característica marcante dos dados. Em termos matriciais, o modelo de análise fatorial pode ser representado por:

$$
X_{p x l}=A_{p x r} F_{r x l}+\varepsilon p x l,
$$

em que: 
$X$ é o vetor de variáveis originais;

$F$ é o vetor de fatores comuns;

$A$ é a matriz de cargas fatoriais;

$\varepsilon$ é o vetor de erros aleatórios;

$r$ é o número de fatores; e

$p$ é o número de variáveis.

Assim, o objetivo do modelo é explicar o comportamento das $p$ variáveis em função de $r$ fatores comuns e de um termo de erro. Além disso, o problema principal é estimar $A$, a matriz de cargas fatoriais. $\mathrm{O}$ método de estimação mais utilizado para estimar as cargas fatoriais, e que também foi adotado neste trabalho, é o de componentes principais, o qual tem como base o uso de raízes características e vetores característicos (Lima, 2015).

Com a finalidade de analisar a adequabilidade dos dados ao método de análise fatorial, alguns testes são propostos, a saber: o teste de esfericidade de Barlett e o critério KMO (Kaiser-Meyer-Olkin). O teste de esfericidade de Barlett verifica se a matriz de correlaçóes é estatisticamente igual a uma matriz identidade, sendo esta a hipótese nula. Então, para que os dados sejam adequados à análise fatorial, deve-se rejeitar a hipótese nula. Quanto ao critério KMO, trata-se de um índice que compara correlaçóes simples e parciais, baseado no princípio de que a matriz de correlaçóes inversa deve ser próxima de uma matriz diagonal, a fim de que a análise fatorial possa ser ajustada aos dados. O critério KMO varia entre 0 e 1 e, segundo indicação da literatura, deve ser maior ou igual a 0,8 para que a análise fatorial seja adequada (Alves et al., 2015).

Autores como Perobelli et al. (1999) e Silva et al. (2014) sugerem um roteiro, que foi adaptado e seguido nesta pesquisa, para a condução da análise fatorial. Inicialmente, é necessário determinar o número de fatores a serem utilizados na análise. Para isso, calculam-se os autovalores da matriz de correlaçóes de tal forma que se mantenha um conjunto de vetores independentes, não correlacionados e que expliquem o máximo da variabilidade dos dados. Segundo Mingoti (2007), dado que os autovalores refletem a importância do fator e que a soma dos autovalores indica a proporçấo da variabilidade dos dados que é explicada, o número de fatores a serem considerados deve explicar em torno de $70 \%$ da variabilidade original dos dados. Outra informação que pode ser extraída dos fatores é a comunalidade, que representa o quanto da variabilidade de cada variável é explicada pelo modelo adotado. O modelo estará mais bem ajustado quanto mais altos forem os valores das comunalidades.

Sequencialmente, após a determinação da quantidade de fatores, estimam-se as cargas fatoriais, que são coeficientes de correlação entre as variáveis e os fatores. 
Como já mencionado, utilizou-se neste trabalho o método de componentes principais para a extração dos fatores.

As cargas fatoriais são úteis para relacionar as variáveis originais com os fatores; entretanto, nem sempre a contribuiçâo das variáveis para cada um dos fatores é suficientemente clara. Assim, para tornar mais nítida a estrutura de relacionamento de variáveis e fatores e facilitar a interpretação, comumente se utiliza o método de rotação de fatores, que permite reorganizar as cargas fatoriais sem, contudo, mudar as comunalidades. O método de rotação mais utilizado, e que também foi adotado neste trabalho, é o varimax, o qual forma um novo sistema de eixos ortogonais, permitindo que grupos de variáveis apareçam com maior nitidez (Lima, 2015).

Como último passo da análise fatorial, calculam-se os escores fatoriais para cada observação. Entre várias aplicaçóes, os escores fatoriais podem ser utilizados para o mapeamento de observaçóes, a criação de índices e a análise de cluster.

Por fim, após os passos da análise fatorial, é criado o IVS, objetivo deste trabalho. Como os escores fatoriais são variáveis padronizadas, eles terão valores positivos e negativos. Assim, para evitar os valores negativos e trazer todos os escores fatoriais para o primeiro quadrante, antes de calcular o índice, procede-se com a seguinte transformação, sugerida por Lima (2015):

$$
F_{i j}^{*}=\frac{F_{i j}-F_{j \min }}{F_{j \max }-F_{\text {min }}}
$$

em que:

$F_{i j}^{*}$ é o escore fatorial transformado do fator $j$ na observaçáo $i$;

$F_{i j}$ é o valor do escore fatorial do fator $j$ na $i$-ésima observação;

$F_{j m a x}$ é o maior valor do $j$-ésimo escore fatorial observado entre todas as observações; e

$F_{j m i n}$ é o menor valor do $j$-ésimo escore fatorial.

A metodologia proposta para o cálculo do IVS leva em consideração esses fatores calculados. Além disso, o novo índice proposto pondera as variáveis pelos valores das raízes características, diferentemente do IVS original, que é resultado de uma média aritmética dos subíndices que englobam variáveis na dimensão de infraestrutura urbana, capital humano e renda e trabalho. Assim, o IVS proposto neste trabalho é calculado a partir de uma média ponderada dos escores fatoriais, em que cada fator recebe um peso correspondente à sua importância na explicação da variância conjunta. Ainda segundo Lima (2015), o índice pode ser definido por:

$$
I V S_{i}=\frac{\sum_{j=1}^{r} \lambda_{j} F_{i j}}{\sum_{j=1}^{r} \lambda_{j}}, i=1,2,3, \ldots, n \mathrm{e} j=1,2,3, \ldots, r
$$


em que:

$I V S_{i}$ é o índice de vulnerabilidade social para a observação $i$;

$\lambda_{j}=$ é a raiz característica relacionada com o fator $j$, isto é, a proporção de variância explicada pelo fator $j$;

$F_{i j}$ é o valor do escore fatorial do fator $j$ na $i$-ésima observação;

$i$ representa observaçóes; e

$j$ representa fatores.

Esse índice proposto, assim como o IVS elaborado pelo Ipea, também varia entre 0 e 1 , sendo que, quanto mais próximo de 1 , maior é a vulnerabilidade social do município. Desse modo, para se poder analisar e confrontar o resultado dos dois índices, os municípios pernambucanos foram classificados de acordo com a seguinte escala:

- 0 a 0,200: muito baixa vulnerabilidade social;

- $\quad 0,201$ a 0,300: baixa vulnerabilidade social;

- $\quad 0,301$ a 0,400: média vulnerabilidade social;

- 0,401 a 0,500: alta vulnerabilidade social; e

- $\quad 0,501$ a 1: muito alta vulnerabilidade social.

Dessa forma, pode-se classificar os municípios do estado de Pernambuco dos mais socialmente vulneráveis até os menos vulneráveis, considerando-se o novo IVS gerado a partir da ferramenta metodológica de análise fatorial.

\section{BASE DE DADOS}

Para operacionalização da estratégia empírica descrita na seção anterior, foram coletados dados do Atlas do Desenvolvimento Humano no Brasil ${ }^{4}$ relacionados às condições de infraestrutura urbana, capital humano e renda e trabalho de todos os 185 municípios pernambucanos no ano de 2010.

Para a construção do índice de vulnerabilidade social aqui proposto, foram utilizados os mesmos indicadores do IVS construído pelo Ipea, e foram adotados os mesmos procedimentos de normalização. A padronização das variáveis é necessária para contornar

40 Atlas do Desenvolvimento Humano no Brasil - desenvolvido pelo Programa das Nações Unidas para o Desenvolvimento (PNUD), Ipea e Fundação João Pinheiro (FJP) - é uma plataforma de consulta a indicadores de demografia, educação, renda, trabalho, habitação e vulnerabilidade, com dados extraídos dos Censos Demográficos de 1991, 2000 e 2010 e abrangência de 5.565 municípios brasileiros, 27 UFs, 21 regiões metropolitanas (RMs) e três regiões integradas de desenvolvimento (Rides) e suas respectivas unidades de desenvolvimento humano (UDHs). Para este trabalho, utilizou-se o escopo dos municípios de Pernambuco, ano de 2010, e os indicadores apresentados na tabela 1. Para mais detalhes, acessar <http://www.atlasbrasil.org.br>. 
o problema de unidades de medidas diferentes e homogeneizar as variâncias. Portanto, cada indicador teve seu valor normalizado numa escala que varia entre 0 e 1 , em que 0 corresponde à situação ideal e 1 corresponde à pior situação. Os indicadores utilizados, bem como os valores de referência da padronização, estão descritos na tabela 1 .

TABELA 1

Variáveis e valores de referência da padronização

\begin{tabular}{|c|c|c|c|}
\hline Variável & Indicadores & $\begin{array}{l}\text { Melhor situação } \\
\quad(0,000)\end{array}$ & $\begin{array}{l}\text { Pior situação }{ }^{1} \\
\quad(1,000)\end{array}$ \\
\hline Ind1 & $\begin{array}{l}\text { Pessoas em domicílios com abastecimento de água e esgotamento } \\
\text { sanitário inadequados (\%) }\end{array}$ & 0,0 & 40,2 \\
\hline Ind2 & População em domicílios sem coleta de lixo (\%) & 0,0 & 50,5 \\
\hline Ind3 & $\begin{array}{l}\text { Vulneráveis que gastam mais de uma hora até o trabalho na } \\
\text { população ocupada vulnerável (\%) }\end{array}$ & 0,0 & 17,8 \\
\hline Ind4 & Mortalidade infantil & 0,0 & 52,5 \\
\hline Ind5 & Crianças de 0 a 5 anos fora da escola (\%) & 0,0 & 98,5 \\
\hline Ind6 & Crianças de 6 a 14 anos fora da escola (\%) & 0,0 & 15,2 \\
\hline Ind7 & Mulheres de 10 a 17 anos que tiveram filhos (\%) & 0,0 & 7,6 \\
\hline Ind8 & $\begin{array}{l}\text { Mães chefes de família sem ensino fundamental e com filho menor, } \\
\text { no total de mães chefes de família (\%) }\end{array}$ & 0,0 & 35,5 \\
\hline Ind9 & Taxa de analfabetismo - 15 anos ou mais & 0,0 & 42,1 \\
\hline Ind10 & $\begin{array}{l}\text { Crianças em domicílios em que ninguém tem ensino fundamental } \\
\text { completo (\%) }\end{array}$ & 0,0 & 87,3 \\
\hline Ind11 & $\begin{array}{l}\text { Pessoas de } 15 \text { a } 24 \text { anos que não estudam, não trabalham e são } \\
\text { vulneráveis, na população dessa faixa (\%) }\end{array}$ & 0,0 & 34,3 \\
\hline Ind12 & $\begin{array}{l}\text { Pessoas com renda domiciliar per capita igual ou inferior a 0,5 } \\
\text { salário mínimo (\%) }\end{array}$ & 0,0 & 100,0 \\
\hline Ind13 & Taxa de desocupação - 18 anos ou mais & 0,0 & 18,0 \\
\hline Ind14 & $\begin{array}{l}\text { Pessoas de } 18 \text { anos ou mais sem ensino fundamental completo e } \\
\text { em ocupação informal (\%) }\end{array}$ & 0,0 & 89,9 \\
\hline Ind15 & Vulneráveis e dependentes de idosos (\%) & 0,0 & 10,5 \\
\hline Ind16 & Taxa de atividade - 10 a 14 anos & 0,0 & 30,1 \\
\hline
\end{tabular}

Fonte: Atlas do Desenvolvimento Humano no Brasil.

Elaboração dos autores.

Nota: ${ }^{1}$ Para mais detalhes sobre o cálculo dos valores de referências, consultar Costa e Marguti (2015).

A ausência de vulnerabilidade social equivale à não ocorrência de casos indesejados, e o valor máximo corresponde à pior situação encontrada a partir da padronizaçáo. Todos os indicadores expostos na tabela 1 apresentam relação direta com situaçóes de vulnerabilidade social. Quanto maior cada um dos indicadores, mais socialmente vulnerável estará o município.

Os resultados da análise são apresentados na próxima seção. 


\section{RESULTADOS}

Primeiramente, investigou-se a adequabilidade dos dados para o uso de análise fatorial por meio do teste de esfericidade de Barlett e do critério KMO. Os resultados dos testes constam da tabela 2.

Como pode ser observado na tabela 2, a hipótese nula do teste de Bartlett foi rejeitada com $99 \%$ de confiança. Isto é, a matriz de correlações é estatisticamente diferente da matriz identidade, o que indica que as correlaçôes entre as variáveis são significativas. Portanto, este resultado sugere que o uso da análise fatorial torna-se pertinente.

Além disso, ainda na tabela 2, observa-se que o valor do critério KMO foi de 0,8237 . A indicação da literatura é que o valor de KMO deve ser igual ou maior que 0,800 para a análise fatorial ser adequada. Dessa forma, pode-se confirmar a consistência dos dados para a análise fatorial.

TABELA 2

Teste de esfericidade de Bartlett e critério KMO

\begin{tabular}{lr}
\hline Testes & \\
\hline Teste KMO & 0,8237 \\
Teste de esfericidade de Barlett & $1.988,17$ \\
Prob (Barlett) & 0,0000 \\
\hline
\end{tabular}

Elaboração dos autores.

Prosseguindo com a análise fatorial, realiza-se a análise com o número de fatores igual ao número de variáveis originais (dezesseis variáveis), de tal modo a se verificar qual o percentual da variabilidade dos dados explicado por cada fator. $\mathrm{Na}$ tabela 3, usando-se o método dos componentes principais, são apresentados os autovalores, o percentual de explicação pertinente a cada fator em relação à variabilidade total e o percentual de variância acumulada.

A decisão quanto ao número de fatores foi feita com base no critério de raiz latente, ${ }^{5}$ o qual recomenda que apenas os fatores com autovalores maiores que 1 devem ser retidos. Seguindo-se este critério, na tabela 3, observa-se que devem ser usados quatro fatores. O primeiro fator explica $36,63 \%$ das variáveis utilizadas, o fator 2 explica 17,65\%, o terceiro fator explica 7,58\% e o fator 4 explica 6,44\%. Assim, o modelo conseguiu explicar aproximadamente $68 \%$ da variância dos dados originais. Apesar de estar um pouco abaixo do valor considerado ideal pela literatura (maior que 70\%), é um valor que pode ser considerado razoável.

5. Para mais detalhes, consultar Kaiser (1960). 
TABELA 3

Autovalores e percentual de variância

\begin{tabular}{lccc}
\hline Fator & Autovalor & Variância $(\%)$ & Variância acumulada (\%) \\
\hline 1 & 5,86150 & 0,3663 & 0,3663 \\
2 & 2,82434 & 0,1765 & 0,5429 \\
3 & 1,21347 & 0,0758 & 0,6187 \\
4 & 1,02996 & 0,0644 & 0,6831 \\
5 & 0,86976 & 0,0544 & 0,7374 \\
6 & 0,80158 & 0,0501 & 0,7875 \\
7 & 0,67479 & 0,0422 & 0,8297 \\
8 & 0,56936 & 0,0356 & 0,8653 \\
9 & 0,51579 & 0,0322 & 0,8975 \\
10 & 0,47058 & 0,0294 & 0,9269 \\
11 & 0,43314 & 0,0271 & 0,9540 \\
12 & 0,27135 & 0,0170 & 0,9710 \\
13 & 0,23137 & 0,0145 & 0,9854 \\
14 & 0,11272 & 0,0070 & 0,9925 \\
15 & 0,06407 & 0,0040 & 0,9965 \\
16 & 0,05621 & 0,0035 & 1,0000 \\
\hline
\end{tabular}

Elaboração dos autores.

No intuito de se verificar o quanto da variabilidade das variáveis originais é explicada pelos quatro fatores comuns do modelo, as comunalidades são apresentadas na tabela 4. Como se pode notar, a maioria das variáveis apresentam comunalidades com valores acima de 0,700 , o que indica forte relação entre as variáveis e os fatores. Assim sendo, além de os resultados dos testes de KMO e Barlett terem sido satisfatórios, a comunalidade também apresentou valores satisfatórios, confirmando a adequabilidade do uso da técnica de análise fatorial.

TABELA 4

\section{Comunalidades das variáveis}

\begin{tabular}{llc}
\hline Variável & & Comunalidades \\
\hline Ind1 & Pessoas em domićlios com abastecimento de água e esgotamento sanitário inadequados (\%) & 0,5542 \\
Ind2 & População em domićlios sem coleta de lixo (\%) & 0,9080 \\
Ind3 & Vulneráveis que gastam mais de uma hora até o trabalho na população ocupada vulnerável (\%) & 0,5073 \\
Ind4 & Mortalidade infantil & 0,4837 \\
Ind5 & Crianças de 0 a 5 anos fora da escola (\%) & 0,5057 \\
\hline
\end{tabular}




\begin{tabular}{|c|c|c|}
\hline Variável & & Comunalidades \\
\hline Ind6 & Crianças de 6 a 14 fora da escola (\%) & 0,3855 \\
\hline Ind7 & Mulheres de 10 a 17 anos que tiveram filhos (\%) & 0,5710 \\
\hline Ind8 & $\begin{array}{l}\text { Mães chefes de família sem ensino fundamental e com filho menor, no total de mães chefes de } \\
\text { família (\%) }\end{array}$ & 0,5407 \\
\hline Ind9 & Taxa de analfabetismo - 15 anos ou mais & 0,8798 \\
\hline Ind10 & Vrianças em domicílios em que ninguém tem ensino fundamental completo (\%) & 0,8075 \\
\hline Ind11 & $\begin{array}{l}\text { Pessoas de } 15 \text { a } 24 \text { anos que não estudam, não trabalham e são vulneráveis, na população dessa } \\
\text { faixa (\%) }\end{array}$ & 0,7965 \\
\hline Ind12 & Pessoas com renda domiciliar per capita igual ou inferior a 0,5 salário mínimo (\%) & 0,8368 \\
\hline Ind13 & Taxa de desocupação - 18 anos ou mais & 0,7921 \\
\hline Ind14 & Pessoas de 18 anos ou mais sem fundamental completo e em ocupação informal (\%) & 0,9116 \\
\hline Ind15 & Vulneráveis e dependentes de idosos (\%) & 0,7300 \\
\hline Ind16 & Taxa de atividade - 10 a 14 anos & 0,7187 \\
\hline
\end{tabular}

Elaboração dos autores.

Seguindo a análise, a etapa seguinte foi relacionar as variáveis com os fatores por meio das cargas fatoriais. A tabela 5 apresenta as cargas fatoriais estimadas para cada fator, isto é, os coeficientes de correlação entre as variáveis e os fatores. Vale ressaltar que, com a finalidade de se melhorar a interpretação dos fatores, os resultados da análise fatorial apresentados na tabela 5 foram elaborados utilizando-se a rotação de eixo pelo varimax.

Na tabela 5, observa-se que, mesmo após o mecanismo de rotação dos fatores, não há uma relação nítida entre as variáveis e os fatores, de modo que se consiga nomeá-los coerentemente. Inicialmente, esperava-se encontrar três fatores que estariam relacionados às mesmas dimensões do índice de vulnerabilidade do Ipea: a dimensão infraestrutura urbana (constituída pelas variáveis Ind1, Ind2 e Ind3), a dimensão capital humano (composta dos indicadores 4 ao 11) e a dimensão renda e trabalho (formada pelos indicadores 12 ao 16). Contudo, o resultado da análise fatorial indicou quatro fatores, e as cargas fatoriais não sugeriram agrupamentos semelhantes ao das classificaçooes das dimensóes no IVS do Ipea. Na verdade, as cargas fatoriais nem ao menos revelaram uma nítida relação sobre qual variável é explicada por qual fator.

Não obstante o resultado das cargas fatoriais encontrado, prosseguiu-se com a análise, objetivando apenas a construção do índice a partir dos escores fatoriais, para então serem classificados os municípios pernambucanos pela ótica da vulnerabilidade social. 
TABELA 5

Distribuição das cargas fatoriais nas variáveis

\begin{tabular}{|c|c|c|c|c|c|}
\hline & Variável & Fator 1 & Fator 2 & Fator 3 & Fator 4 \\
\hline Ind1 & $\begin{array}{l}\text { Pessoas em domicílios com abastecimento de água e esgota- } \\
\text { mento sanitário inadequados (\%) }\end{array}$ & 0,14307 & 0,06824 & $-0,33372$ & 0,13024 \\
\hline Ind2 & População em domicílios sem coleta de lixo (\%) & $-0,06941$ & 0,02646 & $-0,06319$ & 0,84813 \\
\hline Ind3 & $\begin{array}{l}\text { Vulneráveis que gastam mais de uma hora até o trabalho na } \\
\text { população ocupada vulnerável (\%) }\end{array}$ & 0,00411 & $-0,15893$ & 0,14438 & 0,15473 \\
\hline Ind4 & Mortalidade infantil & 0,09730 & 0,04645 & 0,05576 & 0,05954 \\
\hline Ind5 & Crianças de 0 a 5 anos fora da escola (\%) & $-0,02226$ & 0,09357 & 0,29770 & 0,09137 \\
\hline Ind6 & Crianças de 6 a 14 fora da escola (\%) & 0,01181 & $-0,01899$ & 0,34009 & $-0,03921$ \\
\hline Ind7 & Mulheres de 10 a 17 anos que tiveram filhos (\%) & $-0,11411$ & $-0,02367$ & 0,54488 & $-0,08398$ \\
\hline Ind8 & $\begin{array}{l}\text { Mães chefes de família sem ensino fundamental e com filho } \\
\text { menor, no total de mães chefes de família (\%) }\end{array}$ & $-0,07096$ & 0,16865 & 0,23935 & 0,16817 \\
\hline Ind9 & Taxa de analfabetismo - 15 anos ou mais & 0,17625 & 0,05274 & $-0,01942$ & $-0,06869$ \\
\hline Ind10 & $\begin{array}{l}\text { Crianças em domicílios em que ninguém tem ensino funda- } \\
\text { mental completo (\%) }\end{array}$ & 0,07752 & 0,10299 & 0,15444 & $-0,01766$ \\
\hline Ind11 & $\begin{array}{l}\text { Pessoas de } 15 \text { a } 24 \text { anos que não estudam, não trabalham e } \\
\text { são vulneráveis, na população dessa faixa (\%) }\end{array}$ & 0,26051 & $-0,22112$ & $-0,04621$ & 0,02769 \\
\hline Ind12 & $\begin{array}{l}\text { Pessoas com renda domiciliar per capita igual ou inferior a } \\
0,5 \text { salário mínimo }(\%)\end{array}$ & 0,23138 & $-0,04852$ & $-0,08417$ & 0,10092 \\
\hline Ind13 & Taxa de desocupação - 18 anos ou mais & 0,08852 & $-0,25410$ & 0,03796 & 0,05175 \\
\hline Ind14 & $\begin{array}{l}\text { Pessoas de } 18 \text { anos ou mais sem ensino fundamental comple- } \\
\text { to e em ocupação informal (\%) }\end{array}$ & 0,10898 & 0,12879 & $-0,00198$ & $-0,03323$ \\
\hline Ind15 & Vulneráveis e dependentes de idosos (\%) & 0,31399 & $-0,17012$ & $-0,11914$ & $-0,29919$ \\
\hline Ind16 & Taxa de atividade - 10 a 14 anos & $-0,08836$ & 0,25167 & 0,01113 & 0,08597 \\
\hline
\end{tabular}

Elaboração dos autores.

O resultado do índice de vulnerabilidade social calculado neste trabalho, a partir da técnica multivariada de análise fatorial (IVS AF), encontra-se na figura 1. Com a finalidade de comparar os resultados, o IVS desenvolvido pelo Ipea (IVS Ipea) também está representado na figura 1 . O valor médio do IVS AF para os municípios pernambucanos, em 2010, foi de 0,520 , e tem um valor mais alto do que o IVS Ipea, que foi de 0,470 . Isto pode ser visto pela predominância da cor vermelha (faixa de muito alta vulnerabilidade social) no segundo mapa da figura 1. Assim, este resultado sugere que a técnica multivariada de análise fatorial pode ter contribuído para refletir melhor as situaçóes de vulnerabilidade dos municípios pernambucanos e não subestimar a vulnerabilidade social destes. 
FIGURA 1

Mapas do IVS Ipea e do IVS AF dos municípios pernambucanos (2010)

IVS IPEA (2010)

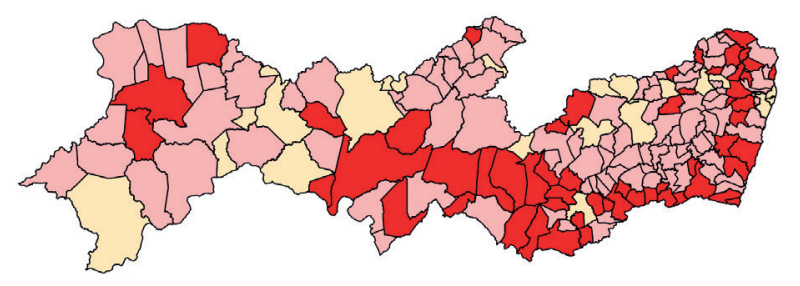

IVS AF (2010)

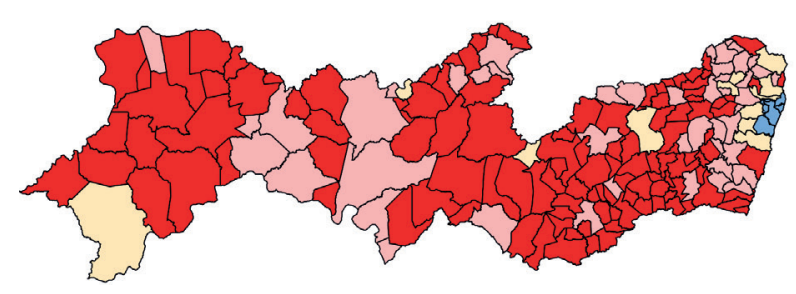

Muito baixa $(0,000-0,200)$

Baixa $(0,201-0,300)$

Média $(0,301-0,400)$

Alta $(0,401-0,500)$

Muito alta $(0,501-1,000)$

Elaboração dos autores.

Pode-se ver na tabela 6 e, de maneira mais ilustrativa, na figura 1 (na qual se comparam a classificação do IVS Ipea e o IVS AF), que, enquanto na classificação do IVS Ipea há apenas 57 municípios considerados com grau de vulnerabilidade social muito alta, no IVS AF essa categoria inclui o maior número de municípios (119, ou seja, $64,3 \%$ do total). No IVS Ipea, a classe de vulnerabilidade que mais engloba os municípios pernambucanos é a classe de alta vulnerabilidade social, que abrange $56,2 \%$ do total de municípios.

Com relação à faixa de muito baixa vulnerabilidade social, não há divergência entre os índices, pois ambos classificaram apenas o distrito estadual de Fernando de Noronha nessa faixa. Por seu turno, na classe de baixa vulnerabilidade, segundo o IVS Ipea, nenhum município aí se enquadrou, ao passo que, pelo IVS AF, são cinco os municípios considerados com baixa vulnerabilidade social (Camaragibe, Jaboatão do Guararapes, Olinda, Paulista e Recife). Assim, pelo IVS AF, como pode ser observado no segundo mapa da figura 1, há uma maior concentração de municípios menos socialmente vulneráveis no litoral pernambucano, mais especificamente na RM de Recife. 


\section{TABELA 6}

Percentual de municípios segundo a classificação do IVS AF e do IVS Ipea

\begin{tabular}{lcccc}
\hline Classificação & $\begin{array}{c}\text { Número de municípios } \\
\text { IVS AF }\end{array}$ & Municípios IVS AF (\%) & $\begin{array}{c}\text { Número de municípios } \\
\text { IVS Ipea }\end{array}$ & $\begin{array}{c}\text { Municípios } \\
\text { IVS Ipea (\%) }\end{array}$ \\
\hline Muito baixo & 1 & 0,5 & 1 & 0,5 \\
Baixo & 5 & 2,7 & 0 & 0,0 \\
Médio & 12 & 6,5 & 23 & 12,4 \\
Alto & 48 & 25,9 & 104 & 56,2 \\
Muito alto & 119 & 64,3 & 57 & 30,8 \\
\hline
\end{tabular}

Elaboração dos autores.

Para complementar a comparação entre os dois índices, na tabela 7 é apresentada uma análise mais agregada, em que é analisado o valor médio dos índices por mesorregiáo pernambucana.

De maneira geral, observou-se que os municípios estão, em média, em pior situação de vulnerabilidade pela classificação do IVS AF do que pela classificação IVS Ipea (conforme a figura 1). Contudo, agregando a análise para as mesorregiôes, na tabela 7 , não se observa um padrão. A RMR, por exemplo, foi a mesorregiáo que apresentou maior discrepância com relação aos dois índices - na classificação da AF, melhorou mais de 0,100 ponto. A mesorregiáo Agreste, por sua vez, apresentou comportamento contrário, estando em pior situação na classificação $\mathrm{AF}$ do que na do Ipea. As demais mesorregiōes praticamente mantiveram o comportamento médio nas duas classificaçóes, excetuando-se a mesorregiáo do Sertáo pernambucano, que se enquadrou na classe de alta vulnerabilidade social pelo IVS Ipea $(0,461)$, e, pela classificaçâoo do IVS AF $(0,540)$, incluiu-se na faixa de muito alta vulnerabilidade social.

TABELA 7

Média dos índices IVS AF e IVS Ipea por mesorregião pernambucana

\begin{tabular}{lccccc}
\hline Indices & Metropolitana & Mata & Agreste & São Francisco & Sertão \\
\hline IVS Ipea & 0,426 & 0,494 & 0,480 & 0,429 & 0,461 \\
IVS AF & 0,327 & 0,492 & 0,573 & 0,492 & 0,540 \\
\hline
\end{tabular}

Elaboração dos autores.

Dessa forma, os resultados apresentados na tabela 7 sugerem que o índice de vulnerabilidade social calculado por meio da técnica de análise fatorial (IVS AF) consegue captar melhor a heterogeneidade das mesorregióes e, consequentemente, dos municípios. No IVS Ipea, as mesorregiôes estão todas agrupadas na faixa de alta vulnerabilidade social. No IVS AF, há maior distinção: a RM de Recife encontra-se na faixa de média vulnerabilidade social; Agreste e Sertão, na faixa de muito alta vulnerabilidade; e Mata e São Francisco, na faixa de alta vulnerabilidade social. 
Após a comparação entre os índices, passa-se agora à análise dos indicadores que os compuseram. As análises serão feitas por mesorregião pernambucana, com a finalidade de se investigar mais detalhadamente o desempenho dos municípios em cada indicador específico. Para isso, os indicadores foram agrupados em três dimensóes. Primeiramente, serão apresentados indicadores relacionados à infraestrutura urbana; em seguida, serão analisados os indicadores relacionados a capital humano; e, por fim, serão considerados os indicadores relativos à situação de renda e trabalho dos municípios.

Vale ressaltar que, assim como o IVS, os indicadores também variam entre 0 e 1 e têm relação direta com a situação de vulnerabilidade. Isto é, quanto mais vulnerável a mesorregião, mais próximo de 1 será o indicador. Indicador com valor igual a 0 significa a ausência de vulnerabilidade para a situação específica que o indicador representa.

TABELA 8

Média dos indicadores de infraestrutura urbana por mesorregião pernambucana

\begin{tabular}{lccccc}
\hline Indicadores & Metropolitana & Mata & Agreste & São Francisco & Sertão \\
\hline $\begin{array}{l}\text { Pessoas em domicílios com abas- } \\
\text { tecimento de água e esgotamento } \\
\text { sanitário inadequados (\%) }\end{array}$ & 0,209 & 0,405 & 0,542 & 0,276 & 0,457 \\
$\begin{array}{l}\text { População em domićlios sem coleta } \\
\text { de lixo (\%) }\end{array}$ & 0,172 & 0,172 & 0,089 & 0,157 & 0,170 \\
$\begin{array}{l}\text { Vulneráveis que gastam mais de uma } \\
\text { hora até } 0 \text { trabalho na população } \\
\text { ocupada vulnerável (\%) }\end{array}$ & 0,811 & 0,489 & 0,276 & 0,291 & 0,259 \\
\hline
\end{tabular}

Elaboração dos autores.

$\mathrm{Na}$ tabela 8, são apresentadas as médias dos indicadores referentes à situação de infraestrutura urbana das mesorregióes pernambucanas. De maneira geral, elas apresentaram, em média, o mesmo comportamento nessa dimensão. Com ressalva para a regiáo metropolitana, que, embora tenha apresentado os menores índices para os demais indicadores dessa dimensão, apresentou o pior resultado no indicador de horas gastas para o trabalho na população vulnerável. Isto reflete o grande inchaço populacional que se verificou na RM de Recife nas últimas décadas segundo Bitoun et al. (2012), em 2010, a região era a quinta mais populosa entre as RMs brasileiras. De fato, essa área cresceu de forma desordenada, sem planejamento e com grandes aglomeraçôes em periferias e favelas, resultando numa precária infraestrutura urbana, especialmente no que se refere à mobilidade urbana. 
TABELA 9

Média dos indicadores de capital humano por mesorregião pernambucana

\begin{tabular}{|c|c|c|c|c|c|}
\hline Indicadores & Metropolitana & Mata & Agreste & São Francisco & Sertão \\
\hline Mortalidade infantil & 0,352 & 0,496 & 0,532 & 0,499 & 0,510 \\
\hline Crianças de 0 a 5 anos fora da escola (\%) & 0,513 & 0,586 & 0,624 & 0,611 & 0,630 \\
\hline Crianças de 6 a 14 fora da escola (\%) & 0,175 & 0,269 & 0,286 & 0,179 & 0,241 \\
\hline Mulheres de 10 a 17 anos que tiveram filhos (\%) & 0,402 & 0,475 & 0,401 & 0,479 & 0,379 \\
\hline $\begin{array}{l}\text { Mães chefes de família sem ensino fundamental e com } \\
\text { filho menor, no total de mães chefes de família (\%) }\end{array}$ & 0,556 & 0,650 & 0,842 & 0,810 & 0,764 \\
\hline Taxa de analfabetismo - 15 anos ou mais & 0,306 & 0,634 & 0,753 & 0,527 & 0,633 \\
\hline $\begin{array}{l}\text { Crianças em domicílios em que ninguém tem ensino } \\
\text { fundamental completo (\%) }\end{array}$ & 0,358 & 0,563 & 0,655 & 0,486 & 0,562 \\
\hline $\begin{array}{l}\text { Pessoas de } 15 \text { a } 24 \text { anos que não estudam, não traba- } \\
\text { Iham e são vulneráveis, na população dessa faixa (\%) }\end{array}$ & 0,542 & 0,775 & 0,607 & 0,600 & 0,689 \\
\hline
\end{tabular}

Elaboração dos autores.

Os indicadores relacionados à dimensão capital humano são expostos na tabela 9. Nesse aspecto, o indicador do percentual de crianças entre 6 e 14 anos fora da escola foi o que apresentou a menor situação de vulnerabilidade, para todas as mesorregiôes pernambucanas. Este resultado pode ser reflexo de políticas sociais como o Programa Bolsa Família - que exigem valores mínimos de frequência escolar para os beneficiários.

\section{TABELA 10}

\section{Média dos indicadores de renda e trabalho por mesorregião pernambucana}

\begin{tabular}{lccccc}
\hline Indicadores & Metropolitana & Mata & Agreste & São Francisco & Sertão \\
\hline $\begin{array}{l}\text { Pessoas com renda domiciliar per capita igual ou } \\
\text { inferior a 0,5 salário mínimo (\%) }\end{array}$ & 0,473 & 0,665 & 0,649 & 0,656 & 0,670 \\
Taxa de desocupação - 18 anos ou mais & 0,795 & 0,735 & 0,342 & 0,487 & 0,405 \\
$\begin{array}{l}\text { Pessoas de } 18 \text { anos ou mais sem ensino fundamental } \\
\text { completo e em ocupação informal (\%) }\end{array}$ & 0,420 & 0,614 & 0,754 & 0,627 & 0,699 \\
Vulneráveis e dependentes de idosos (\%) & 0,365 & 0,609 & 0,552 & 0,432 & 0,522 \\
Taxa de atividade - 10 a 14 anos & 0,137 & 0,164 & 0,463 & 0,382 & 0,417 \\
\hline
\end{tabular}

Elaboração dos autores.

Por fim, na tabela 10, encontra-se o desempenho médio das mesorregióes pernambucanas nos indicadores do âmbito de renda e trabalho. Nessa dimensão, a mesorregiáo do Agreste chama a atenção por apresentar a menor vulnerabilidade para o indicador da taxa de desocupação, refletindo o aquecimento da economia da região, caracterizada principalmente pelo polo de confecçóes.

Contudo, os dados da tabela 10 também revelam que a mesorregiáo do Agreste apresenta o maior percentual de pessoas empregadas em ocupação informal, o que é 
reflexo do alto grau de informalidade da principal atividade econômica da região o setor têxtil. Além disso, a taxa de atividade entre indivíduos na faixa etária entre 10 e 14 anos é maior nessa mesorregião. Assim, apesar de aquecer o mercado de trabalho, há indícios de que o polo de confecçôes do Agreste pernambucano é caracterizado pela informalidade e pelo uso de mão de obra infantil.

Longe de esgotar a literatura sobre o tema em questáo, este trabalho propôs uma forma alternativa de cálculo do índice de vulnerabilidade social para os municípios pernambucanos.

\section{CONSIDERAÇÕES FINAIS}

Este trabalho teve como objetivo classificar os municípios pernambucanos pelo índice de vulnerabilidade social construído, para o ano de 2010, a partir da técnica multivariada de análise fatorial. Além disso, o estudo comparou os resultados do IVS calculado pela análise fatorial com os do IVS já estabelecido pelo Ipea.

Em termos gerais, a partir da análise, observou-se que o valor médio do IVS AF para os municípios pernambucanos, em 2010, foi de 0,520 , e teve um valor mais alto do que o IVS Ipea, que foi de 0,470. Assim, este resultado sugere que a técnica multivariada de análise fatorial pode ter contribuído para refletir melhor as situaçôes de vulnerabilidade dos municípios pernambucanos e não subestimar a vulnerabilidade social destes.

Com relação aos resultados, observou-se que, na classificação de ambos os índices, o distrito estadual de Fernando de Noronha foi a única localidade pernambucana situada na faixa de muito baixa vulnerabilidade social. Pelo IVS AF, notou-se uma maior concentração de municípios menos socialmente vulneráveis no litoral pernambucano, mais especificamente na RM de Recife. Ainda segundo o índice desenvolvido pela técnica de análise fatorial, constatou-se que 64,3\% dos municípios pernambucanos apresentam muito alta vulnerabilidade social.

Dessa forma, o trabalho permite uma observação mais acurada dos municípios pernambucanos e sinaliza a atenção para os municípios mais vulneráveis, no sentido de se emitir um alerta para os gestores de políticas públicas. Com o resultado, podem-se focalizar políticas públicas no intuito de mitigar as vulnerabilidades dos municípios que apresentaram os piores índices.

Não obstante, o trabalho pode ser aprimorado em vários aspectos. Como sugestôes para trabalhos futuros, pode-se ampliar o cálculo do índice para todos os municípios brasileiros, acrescentar variáveis aos indicadores, bem como calcular o índice para o ano 2000 e observar a evoluçáo dos municípios. 


\section{REFERÊNCIAS}

ALVES, F. F.; ALVES, J. H.; LIMA, J. E.IDH-Revisitado: uma abordagem alternativa para mensurar a real situação do desenvolvimento municipal. Revista Eletrônica Documento/Monumento, v. 14, p. 424-447, 2015.

BALAN, M. A multidimensional analysis of social vulnerability. Hyperion Economic Journal, v. 3, n. 1, 2013

BITOUN, J. et al. Região metropolitana do Recife no contexto de Pernambuco no Censo 2010. Recife: Observatório das Metrópoles, 2012.

COSTA, M. A.; MARGUTI, B. O. Atlas da vulnerabilidade social nos municípios brasileiros. Brasília: Ipea, 2015.

KAISER, H. F. The application of electronic computers to factor analysis. Educational and Psychological Measurement, v. 20, p. 141-151, 1960.

LIMA, J. E. Curso de análise estatística multivariada. Viçosa: UFV, 2015.

MINGOTI, S. A. Análise de dados através de métodos de estatística multivariada: uma abordagem aplicada. Belo Horizonte: UFMG, 2007.

PEROBELLI, F. S.et al. Planejamento regional e potenciais de desenvolvimento dos municípios de Minas Gerais na região em torno de Juiz de Fora: uma aplicação da análise fatorial. Nova Economia, Belo Horizonte, v. 9, n. 1, p. 121-150, 1999.

PLESA, R. Social exclusion and vulnerable populations. Annals of the University of Petroşani, Economics, v. 13, n. 2, p. 173-180, 2013.

SILVA, N. C. N. et al. O uso da análise fatorial na descrição e identificação dos perfis característicos de municípios de Minas Gerais. Rev. Bras. Biom., São Paulo, v. 32, n. 2, p. 201-215, 2014.

Data da submissão: 13/9/2017

Primeira decisão editorial em: 4/6/2018

Aprovação final em: 4/6/2018 